\title{
Elevation of miR-125b-5p is related to improved prognosis in laryngeal squamous cell carcinoma and inhibits the malignancy and glycometabolic disorder by targeting MAP3K9
}

\author{
Fu CHEN ${ }^{1, *}$, Zheng LAO $^{2}$, Hai-Yan ZHANG ${ }^{1}$, Jie WANG ${ }^{1}$, Sheng-Zi WANG ${ }^{1, *}$ \\ ${ }^{1}$ Department of Radiation Oncology, Eye and ENT Hospital of Fudan University, Shanghai, China; ${ }^{2}$ Radiotherapy Division, Department of Oral \\ and Maxillofacial-Head and Neck Oncology, Ninth People's Hospital Affiliated to Shanghai Jiao Tong University School of Medicine, Shanghai, \\ China
}

*Correspondence: fuchen@fudan.edu.cn; shengziwang@fudan.edu.cn

Received October 27, 2021 / Accepted January 17, 2022

\begin{abstract}
Laryngeal squamous cell carcinoma (LSCC) is the most common malignant tumor in the head and neck cancer, with a poor prognosis. As we know, microRNAs (miRNAs) play a vital role in the initiation and development of various cancers including LSCC. In this study, we explored the role of miR-125b-5p and its downstream regulatory pathway in LSCC. Our data demonstrated that miR-125b-5p expression was significantly downregulated in LSCC tissues and cells. LSCC patients with high expression of miR-125b-5p had higher overall survival (OS) and were closely related to the clinical stage. Overexpression of miR-125b-5p impaired viability and glycolysis, and facilitated apoptosis in LSCC cells. And miR-125b-5p silencing had the opposite effects. Bioinformatics website predicted that MAP3K9 was one of the potential target genes of miR-125b-5p. Cell experiments demonstrated that miR-125b-5p repressed the MAP3K9 levels by directly targeting MAP3K9. Additionally, the negative correlation between miR-125b-5p and MAP3K9 was validated in LSCC tissues. Overexpression of MAP3K9 attenuated the inhibitory effect of miR-125b-5p on viability and glycolysis, and the pro-apoptosis effect of miR-125b-5p in LSCC cells. Furthermore, in vivo experiments demonstrated that tumor growth was hampered in AMC-HN-8 cells transfected with miR-125b-5p mimic. In contrast, the knockdown of miR-125b-5p reduced tumor growth in vivo. Meanwhile, the in vivo immunohistochemistry and TUNEL assays suggested that the miR-125b-5p overexpression restrained cell proliferation and promoted apoptosis via targeting MAP3K9. Overall, these above results suggested that miR-125b-5p suppressed proliferation and glycolysis, and promoted apoptosis by directly targeting MAP3K9 in LSCC cells. Thus, miR-125b-5p acts as a tumor suppressor miRNA and the miR-125b-5p/MAP3K9 axis may be a promising candidate for LSCC treatment.
\end{abstract}

Key words: laryngeal squamous cell carcinoma; miR-125b-5p; MAP3K9; proliferation; apoptosis; glycolysis

Squamous cell carcinoma accounts for more than $90 \%$ of head-and-neck cancer, which is the seventh most frequent cancer in the world [1]. And these tumors were commonly found in the oral cavity, oropharynx, hypopharynx, and larynx $[2,3]$. Notably, as the most prevalent form of laryngeal cancer, laryngeal squamous cell carcinoma (LSCC) constitutes around $25 \%$ of head and neck carcinoma worldwide [4]. Currently, treatments for LSCC include surgical resection, radiotherapy, and chemotherapy [3]. Due to the lack of diagnostic biomarkers for the early-stage LSCC, most diagnosed cases are at the advanced stage with metastasis [5]. Although great progress has been made to improve the management of LSCC, the treatment of LSCC remains challenging [6]. Therefore, it's essential to have a better understanding of the initiation and progression of LSCC and find novel predictive biomarkers and therapeutic targets in LSCC therapy.

As is well known, microRNAs (miRNAs) are recently discovered non-coding small RNA molecules, which could decrease the target gene levels and participate in the development of many kinds of malignant tumors $[7,8]$. Studies have shown that miRNAs account for only $2 \%$ of the total number of human genes, whereas they regulate more than $30 \%$ of human gene expression, including cell growth and this process involves cell growth, differentiation, apoptosis, and other biological functions $[9,10]$. Focusing on human laryngeal cancer, a variety of studies demonstrated that miRNAs are differentially expressed in laryngeal carcinoma to exert 
a carcinogenic or anti-carcinogenic role [11]. For instance, Shuang et al. reported that miR-503 was found to be reduced in LSCC, which may play the role of proto-oncogenes by directly targeting PDCD4 to promote tumor growth [12]. Another study confirmed that miR-204-5p repressed the metastasis of LSCC cells through modulating FOXC1, indicating that miR-204-5p has the potential to become a biological target for LSCC treatment [13]. The miR-125b-5p discussed in this study was widely expressed in the body and participated in the regulation of tumor cell growth and the progression of nerve injury $[14,15]$. In cancer, miR-125b-5p was demonstrated to be elevated in breast cancer (BC) cells and could reduce the tumor cell growth by regulating KIAA1522 [16]. In addition, miR-125b-5p restrained the metastasis of hepatocellular carcinoma (HCC) cells by downregulating TXNRD1 [17]. It is worth noting that one previous study discovered that miR-125b-5p impaired cell proliferation by inhibiting glycolysis in LSCC [18]. However, there are still few studies on miR-125b-5p in LSCC, and more related studies are required to comprehensively elaborate its functional roles in LSCC.

In the present study, the clinical significance and downstream mechanism of miR-125b-5p in LSCC were investigated through various experiments, including CCK-8, clone formation, flow cytometry, luciferase reporter, xenograft tumor, TUNEL, and immunohistochemical assays. These data suggested that miR-125b-5p plays a role in suppressing cancer in LSCC, which might be a potential diagnostic biomarker.

\section{Patients and methods}

Clinical samples. LSCC tissues and paired normal tissues were collected from 29 LSCC patients at Eye and ENT Hospital of Fudan University. After collection, the tissue samples were immediately soaked in RNAfixer solution (BioTeke, Beijing, China), and then transferred to the refrigerator at $-80^{\circ} \mathrm{C}$. This study was based on the principles expressed in Helsinki Declaration. All clinical experiments were approved by the Ethics Committee of the Eye and ENT Hospital of Fudan University Participants provided written informed consent to participate in the study and consent for publication was obtained from all participants.

Cell culture. Two LSCC cell lines (AMC-HN-8 and Tu686) and human normal bronchial epidermal cells $(16 \mathrm{HBE})$ were obtained from the National Infrastructure of Cell Line Resource (Beijing, China). All cells were kept in DMEM (Thermo Fisher Scientific, Inc., USA) supplemented with $10 \%$ fetal bovine serum (FBS; Sigma-Aldrich, USA) in a $5 \% \mathrm{CO}_{2}$ incubator at $37^{\circ} \mathrm{C}$. Cells in logarithmic phase cells were taken for follow-up experimental study.

qRT-PCR. Total RNA was isolated from LSCC tissues, normal tissues, and cells by TRIzol reagent. RNA samples were reverse transcribed into cDNA through the reverse transcription kit (Promega). Subsequently, PCR was performed on a 7500 real-time PCR system (Applied Biosystems) using SYBR Green PCR kit (Biotech, China). GAPDH and U6 were detected as internal control and data were calculated using the $2^{-\Delta \Delta} \mathrm{Ct}$ method [19]. According to the previous studies [20, 21 ], the primers were listed as followed: miR-125b-5p forward 5'-TCCCTGAGACCCTAACTTGTGA-3' and miR-125b-5p reverse 5'-AGTCTCAGGGTCCGAGGTATTC-3'; MAP3K9 forward 5'-GAGTGCGGCAGGGACGTAT-3' and MAP3K9 reverse 5'-CCCCATAGCTCCACACATCAC-3'; U6 forward 5'-TGCGGGTGCTCGCTTCGGCAGC-3' and U6 reverse 5'-CCAGTGCAGGGTCCGAGGT-3'; GAPDH forward 5'-GAAGGTGAAGGTCGGAGTC-3' and GAPDH reverse 5'-GAAGATGGTGATGGGATTTC-3'. The 2-step PCRs were performed as followed: $95^{\circ} \mathrm{C}$ for $2 \mathrm{~min}, 40$ cycles of $95^{\circ} \mathrm{C}(30 \mathrm{~s}), 60^{\circ} \mathrm{C}(50 \mathrm{~s})$, and $72^{\circ} \mathrm{C}(30 \mathrm{~min})$.

Cell transfection. miR-125b-5p overexpression plasmid (miR-125b-5p\#1 and -\#2) and their control (miR-NC), miR-125b-5p inhibitor (anti-miR-125b-5p), and corresponding negative control (anti-NC) were obtained from the GenePharma Company. The pcDNA-MAP3K9 expression plasmids (MAP3K9) and empty vector ( $\mathrm{pcDNA}$ ) were purchased from ViGene Biosciences (China). LSCC cells were incubated into 6-well plates and cultured at the confluence of $50 \%$ prior to transfection. Next, these above plasmids were transfected into LSCC cells and Lipofectamine 2000 (Invitrogen) was used in the transfection procedure. Cells were taken for the subsequent experimental studies after $48 \mathrm{~h}$.

CCK-8 assay. Cells (800 cells/well) were seeded in 96-well plates and cultured for the indicated time. CCK- 8 solution ( $10 \mu \mathrm{l} /$ well) was added to the cells at 24,48 , and $72 \mathrm{~h}$ posttreatment. Absorbance at $450 \mathrm{~nm}$ was detected with a microplate reader (BioTek) after $2 \mathrm{~h}$ incubation in the dark.

Colony formation assay. Cells (500 cells/well) were seeded into 6-well plates and cultured for 2 weeks at $37^{\circ} \mathrm{C}$. Subsequently, $4 \%$ methanol and $0.1 \%$ crystal violet were performed to fix and stain the cells, respectively. At the completion of staining, cells were washed with running tap water, and the number of colonies formed was counted with the Image J software (NIH).

Flow cytometry analysis. According to the manufacturer's guidelines, Annexin V-FITC Apoptosis Detection Kit (Sigma-Aldrich) was used to assess the cell apoptosis. Briefly, cells were treated as indicated and harvested by centrifugation at $5,000 \times \mathrm{g}$ for $3 \mathrm{~min}$, followed by pre-cold PBS washed twice. Next, the cells were resuspended in the $1 \times$ annexin binding buffer. Then $5 \mu \mathrm{l}$ of PI and $5 \mu \mathrm{l}$ of Annexin V were added to the suspension and mixed the solution and cells gently. After incubating in the dark for $20 \mathrm{~min}$, flow cytometry was conducted to determine the apoptosis.

Glucose uptake and lactate production. Glucose uptake and lactate production were measured using enzyme-linked immunosorbent assays (ELISA). Briefly, the lactate production and glucose consumption were assessed by the lactate colorimetric assay kit and glucose assay kit (Biovision, USA), respectively, as previously described [18]. 
Western blot. Cells with indicated treatment or tissues were lysed on ice using RIPA buffer (Beyotime, China). Total proteins were quantified by the Bradford assay (Beyotime, China) following the manufacturer's guidelines. Protein samples were separated on SDS-PAGE and then transferred to the PVDF membrane (Beyotime, China). These membranes were blocked in 5\% nonfat milk for $1 \mathrm{~h}$, followed by the incubation with the primary antibodies (dilution $1: 1000)$ and then these membranes were incubated with a secondary antibody (dilution 1:5000) for $3 \mathrm{~h}$. These protein bands were visualized with chemiluminescence substrate (Beyotime, China). Primary and secondary antibodies are listed as follows: anti-HK2 primary antibody (cat. no. \#2867, Cell Signaling Technology, USA), anti-MAP3K9 primary antibody (cat. no. ab154506, Abcam, USA), anti-GAPDH primary antibody (cat. no. \#5174, Cell Signaling Technology, USA), anti-HRP conjugated goat anti-rabbit secondary antibody (cat. no. ab6721, Abcam, USA).

Luciferase reporter assay. Cells were incubated in a 24-well plate at 70\% confluency and were co-transfected with miR-NC or miR-125b-5p mimics, and plasmids harboring either wild-type (WT) or mutated 3'-untranslated regions (UTR) of MAP3K9 using Lipofectamine 2000. After $48 \mathrm{~h}$ of transfection, the luciferase kit (Promega, USA) was employed to determine the luciferase activities.

Immunocytochemistry. Tumor slices were fixed in formaldehyde and then embedded in paraffin. Antigen retrieval was performed by using a water bath heated to $90{ }^{\circ} \mathrm{C}$ for $30 \mathrm{~min}$, followed by anti-Ki-67 primary antibody (1:1000; cat. no. ab16667, Abcam, USA) and co-incubation overnight at $4{ }^{\circ} \mathrm{C}$. After washing, slices were stained with the HRP-labeled secondary antibody (1:5000; cat. no. LK-GAR007, Abcam) for $30 \mathrm{~min}$. Finally, DAB was added to develop the color. Ki-67 was located in the nucleus, and the nuclei stained with brown-yellow were considered to be positive.

TUNEL staining. TUNEL apoptosis kit (Roche, Basel, Switzerland) was conducted to assess apoptosis following the manufacturer's guidelines. In short, tumor tissue sections were incubated with fluorescein (green)-labeled dUTP solution at $37^{\circ} \mathrm{C}$ for $1 \mathrm{~h}$, and photographed under the BX64 fluorescence microscope (Olympus, Japan). The number of positive cells (green) in the fluorescent image represents apoptosis.

In vivo experiment. Twelve $\mathrm{BALB} / \mathrm{c}$ mice were purchased from Slyke Jingda Company and randomly divided into two groups. For the experimental group $(n=6)$, AMC-HN- 8 cells, which were stably transfected with miR-125b-5p overexpression or knockdown lentivirus plasmids, were subcutaneously injected into both flanks of each mouse. As for the control group $(n=6)$, AMC-HN-8 cells transfected with control lentivirus were injected subcutaneously. The tumor size was monitored by calipers for about 27 or 22 days, respectively. After inoculation, tumor tissues were removed from the mice and weighed. Tumors were subjected to analysis by qRT-PCR and western blotting. The remaining tumor tissues were refrigerated for a follow-up experimental study. Animal experiments were performed in accordance with the National Institutes of Health (NIH) guidelines.

Statistical analysis. All data in the present study were analyzed using GraphPad Prism 8 (GraphPad 8, USA). Quantitative data are presented as mean \pm standard deviation (SD). Student's t-tests were used to identify the significance in cell experiments, and one-way ANOVA was performed to determine the $\mathrm{p}$ value between different groups followed by a Tukey post hoc test. Kaplan-Meier (K-M) curves were performed to exhibit overall survival (OS) in LSCC patients and Log-Rank was carried out to evaluate the difference between the two groups. If $\mathrm{p}<0.05$, the differences were considered significant.

\section{Results}

miR-125b-5p expression was significantly reduced in LSCC tissues and cells. We first studied the expression of miR-125b-5p in LSCC, as shown in Figure 1A, through the GEO database (GEO: GSE116994) (https://www.ncbi. nlm.nih.gov/geo/) analysis. miR-125b-5p expression was downregulated in LSCC tissues compared to control. Meanwhile, in our study, compared with normal tissues, miR-125b-5p expression in LSCC tissues was remarkably decreased (Figure 1B). Moreover, miR-125b-5p levels of both LSCC cell lines were significantly lower than those in normal cells (Figure 1C). In addition, we also analyzed the clinical relevance of miR-125b-5p in LSCC. Low expression of miR-125b-5p could bring a better prognosis to patients with LSCC (Figure 1D). In clinical aspects, the lower miR-125b-5p level was significantly related to the clinical stage, but not age, gender, lymph node metastasis, and differentiation (Table 1). The above results indicated the decreased miR-125b-5p expression may impair LSCC progression.

miR-125-5p served as a tumor suppressor role in LSCC cell growth and glycolysis. To understand the specific effect of miR-125b-5p in LSCC, miR-125b-5p mimic was introduced into AMC-HN-8 and Tu686 cells. As presented in Figure 2A, miR-125b-5p expression in the miR-125b-5p\#2 group was highest compared with other groups, suggesting a successful transfection, and miR-125b-5p\#2 was selected for the next study. Then, the effect of miR-125b-5p on cell proliferation was investigated by the CCK- 8 assay. The viability of both AMC-HN-8 and Tu686 cells was lower in the miR-125b\#2 group than that of the miR-NC group (Figures 2B, 2C). The flow cytometry and colony formation assay further confirmed the suppression effect of miR-125b-5p in LSCC. As shown in Figure 2D, the overexpression of miR-125b-5p significantly enhanced the apoptosis of AMC-HN-8 and Tu686 cells. Likewise, the clonogenicity of miR-125b-5p overexpressing AMC-HN-8 and Tu686 cells decreased significantly (Figure 2E). Subsequently, lactate production and glucose uptake were measured by ELISA 


\section{A GSE117007/GPL19117/MIMRT0000423_st}
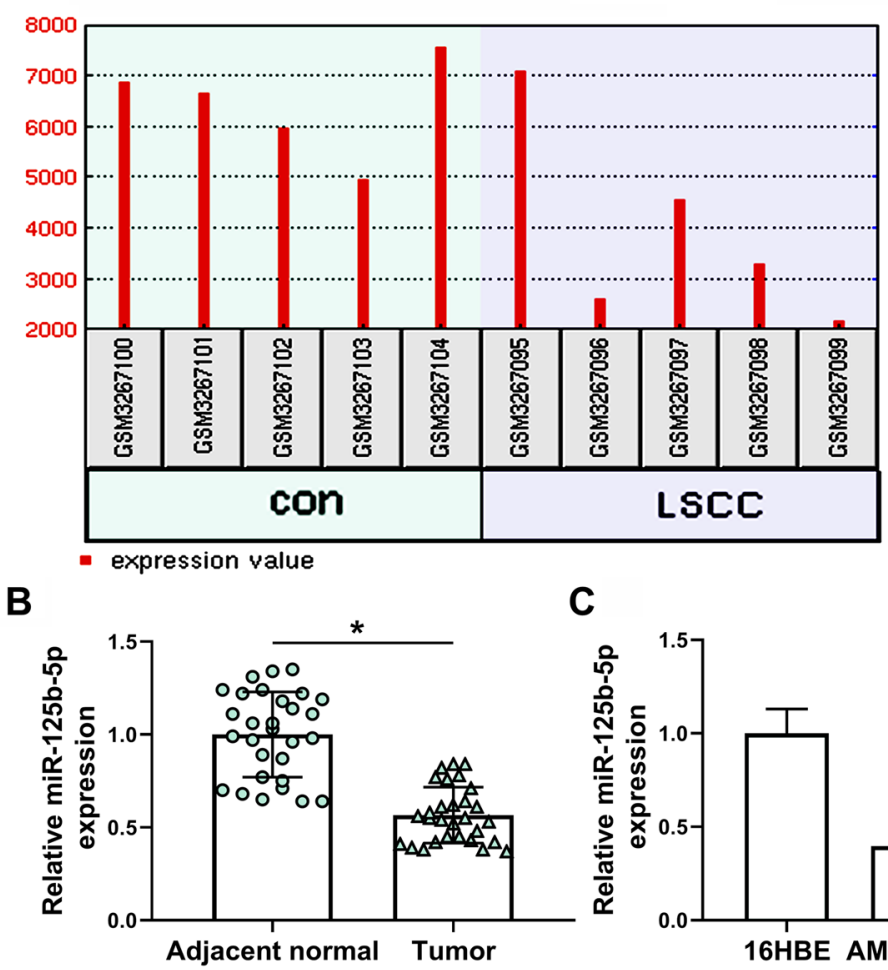

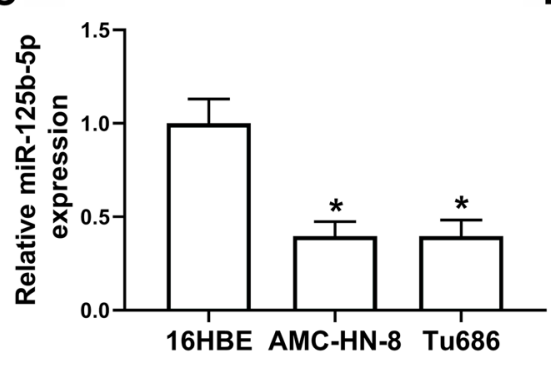

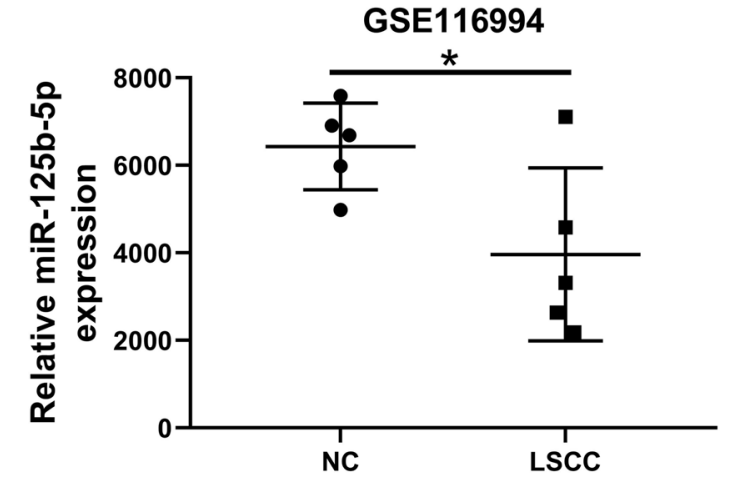

- High miR-125b-5p expression

D

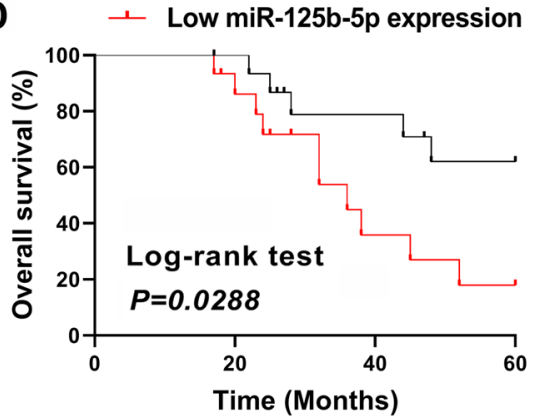

Figure 1. miR-125b-5p expression was significantly reduced in LSCC tissues and cells. A) The data set of miR-125b-5p expression in LSCC from the Gene Expression Omnibus (GEO) database. B) The expression of miR-125b-5p in LSCC tissues $(n=29)$ and corresponding adjacent normal tissues $(n=29)$ was measured by qRT-PCR. ${ }^{\star} \mathbf{p}<0.05$. C) Expression of miR-125b-5p in LSCC cells (AMC-HN-8 and Tu686) and normal cell (16HBE) was measured by qRT-PCR. ${ }^{*}$ p $<0.05$ vs. 16 HBE. D) Kaplan-Meier analysis of overall survival of LSCC patients with high vs. low expression of miR-125b-5p.

assay to evaluate the effect of miR-125b-5p on glycolysis. As shown in Figures $2 \mathrm{~F}$ and $2 \mathrm{G}$, the overexpression of miR-125-5p significantly reduced lactate production and glucose uptake in the miR-125b-5p\#2 group compared with the miR-NC group. Hexokinase 2 (HK2), an essential enzyme in glucose metabolism, was detected by western blotting. Results showed that miR-125b-5p overexpression markedly downregulated the protein level of HK2 in both AMC-HN-8 and Tu686 cells (Figure 2H). In addition, the expression of miR-125b-5p was knocked down in AMC-HN-8 and Tu686 cells. As shown in Figure 2I, the expression of miR-125b-5p in the anti-NC group was significantly lower than that in the anti-miR-125b-5p group. Results of the CCK-8 assay showed that knockdown of miR-125b-5p significantly promoted the proliferation of AMC-HN-8 and Tu686 cells (Figure 2J). Meanwhile, miR-125b-5p knockdown significantly increased the protein expression of HK2 (Figure $2 \mathrm{~K}$ ). To sum up, these findings indicated that miR-125b-5p overexpression suppressed the growth and glycolysis of LSCC cells, and miR-125b-5p knockdown had the opposite effects.

MAP3K9 was the direct target of miR-125b-5p. To further understand the inhibitory mechanism of
miR-125b-5p on LSCC cell proliferation and glycolysis, the downstream targets of miR-125b-5p were predicted by using online tools (ENCORI and miRDB). The predicted results revealed MAP3K9 was one of the potential targets of miR-125b-5p (Figure 3A). Only one study reported that miR-125b-5p promoted the progression of LSCC by targeting TRIB2 [22]. MAP3K9 plays a tumor-promoting role in various tumors except for LSCC. However, there is no research regarding the expression and regulatory mechanism of MAP3K9 in LSCC. Therefore, through comprehensive analysis, we finally determined MAP3K9 as the target gene of miR-125b-5p in the present study. Then, the luciferase analysis was deployed to verify the relationship between miR-12b-5p and MAP3K9. AMC-HN-8 and Tu686 cells were co-transfected with plasmids containing MAP3K9-WT or Mut and miR-125b-5p mimic or miR-NC. In AMC-HN-8 and Tu686 cells co-transfected with MAP3K9 3'-UTR WT and miR-125b-5p mimic, we found that the luciferase activity was significantly decreased, but the luciferase activity remained unchanged in cells co-transfected with MAP3K9 3'-UTR Mut and miR-125b-5p mimic (Figures 3B, 3C). Next, MAP3K9 expression in LSCC tissues 

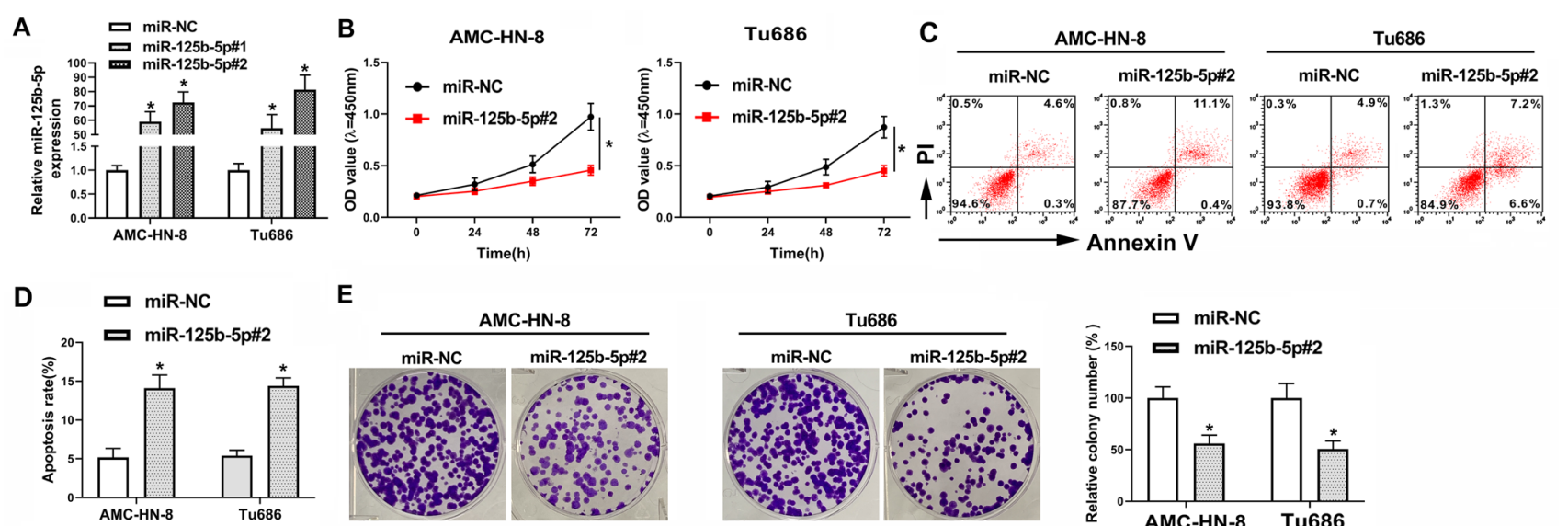

$\mathrm{E}$
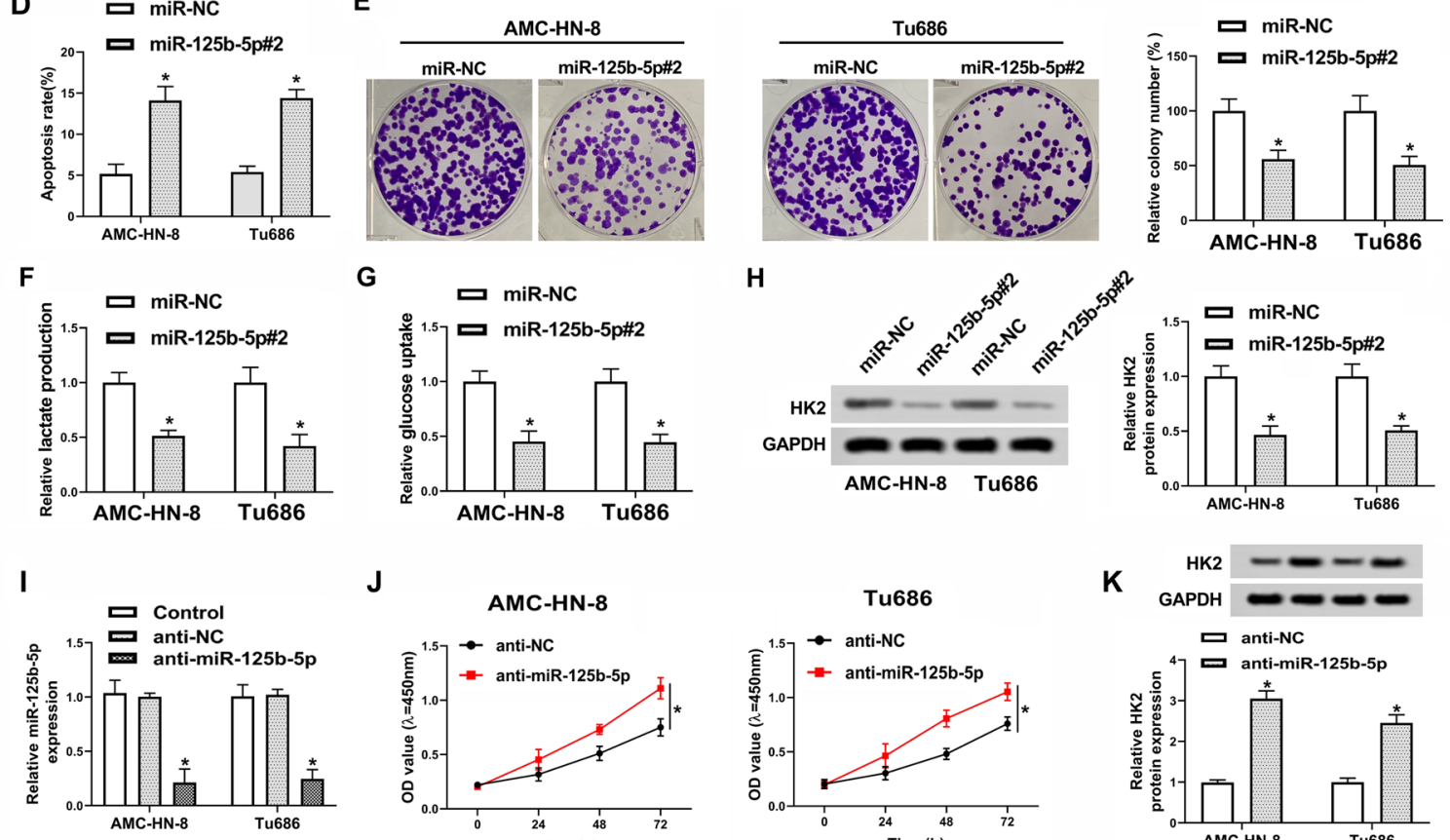

J
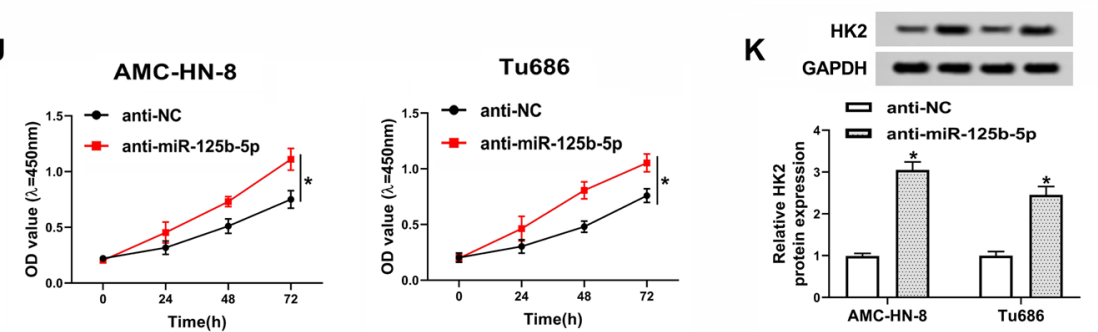

Figure 2. miR-125-5p served as a tumor suppressor role in LSCC cell growth and glycolysis. A) The efficiency of miR-125b-5p overexpression was measured by qRT-PCR after $48 \mathrm{~h}$ of transfection. B) Cell viability was assessed by the CCK-8 assay in AMC-HN-8 cells transfected with miR-NC or miR125b-5p\#2. C) Cell viability was assessed by the CCK-8 assay in Tu686 cells transfected with miR-NC or miR-125b-5p\#2. D) Apoptosis was detected by flow cytometry assay. E) The relative number of clones was measured by colony formation assay in AMC-HN-8 and Tu686 cells transfected with miR-NC or miR-125b-5p\#2. F) Lactate production was measured by ELISA assay. G) Glucose uptake was measured by ELISA assay. H) The expression of HK2 was measured by western blotting in AMC-HN-8 and Tu686 cells transfected with miR-NC or miR-125b-5p\#2. ${ }^{*}$ p $<0.05$ vs. miR-NC. I) The efficiency of miR-125b-5p knockdown was measured by qRT-PCR after $48 \mathrm{~h}$ of transfection. ${ }^{\star}$ p $<0.05$ vs. anti-NC. J) Cell viability was assessed by the CCK-8 assay in AMC-HN-8 cells transfected with anti-NC or anti-miR-125b-5p. ${ }^{\star} \mathrm{p}<0.05$ vs. anti-NC. K) The expression of $\mathrm{HK} 2$ was measured by western blotting in AMC-HN-8 and Tu686 cells transfected with anti-NC or anti-miR-125b-5p. ${ }^{\star}$ p $<0.05$ vs. anti-NC.

and cells was evaluated by qRT-PCR and western blotting. Compared with the normal tissues, the mRNA and protein levels of MAP3K9 in LSCC tissues were significantly upregulated (Figures 3D, 3E). Besides, compared with the $16 \mathrm{HBE}$ cells, the mRNA and protein levels of MAP3K9 in both cells were also increased significantly (Figures 3F, 3G). Moreover, correlation analysis of 29 LSCC samples showed that miR-125b-5p could negatively modulate MAP3K9 expression (Figure $3 \mathrm{H}$ ). To sum up, these data hinted that miR-125b-5p directly targeted MAP3K9 and negatively regulated its expression in LSCC.

miR-125b-5p inhibited LSCC cell growth and glycolysis by downregulating MAP3K9. Based on these above findings, we hypothesized that MAP3K9 may mediate the effect of miR-125b-5p in LSCC. To verify the hypothesis, the overexpression plasmid of MAP3K9 (pcDNA-MAP3K9) was synthesized, and the transfection efficiency was detected by qRT-PCR and western blotting. As presented in Figures 4A and $4 \mathrm{~B}$, overexpression of miR-125b-5p significantly reduced MAP3K9 expression in both cells, and these changes were partially reversed by MAP3K9 overexpression, suggesting a successful transfection. CCK-8 assay, apoptosis, and colony formation assays revealed miR-125b-5p overexpression remarkably inhibited AMC-HN-8 and Tu686 cell proliferation while promoting apoptosis, which was all alleviated by the overexpression of MAP3K9 (Figures 4C-4F). In addition, the glycolysis was assessed by ELISA and western blotting to determine glucose uptake, lactate production, and HK2 expression. Results showed the inhibition of miR-125b-5p overexpression on glucose uptake, lactate production, and 
A

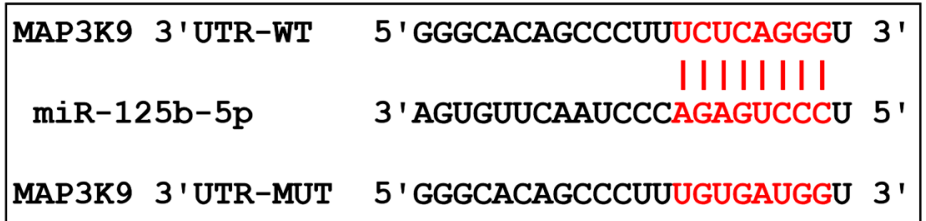

B

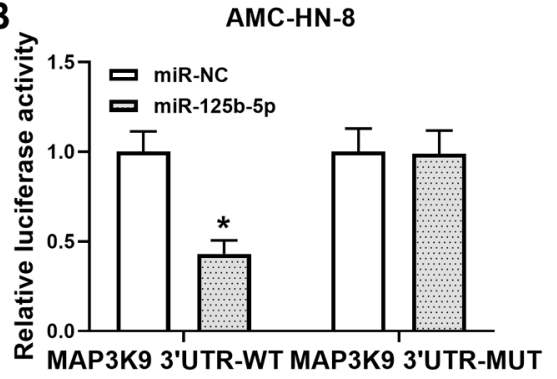

C

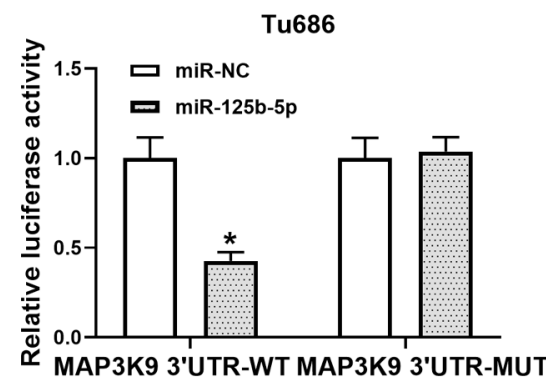

D
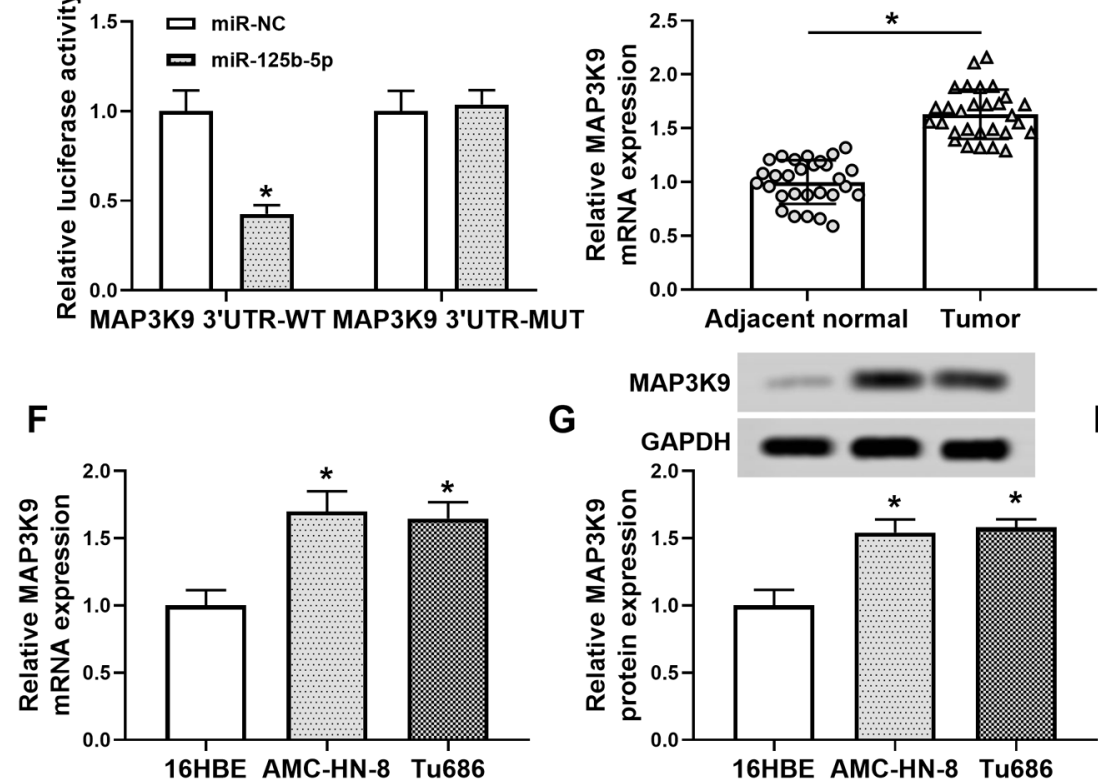

G

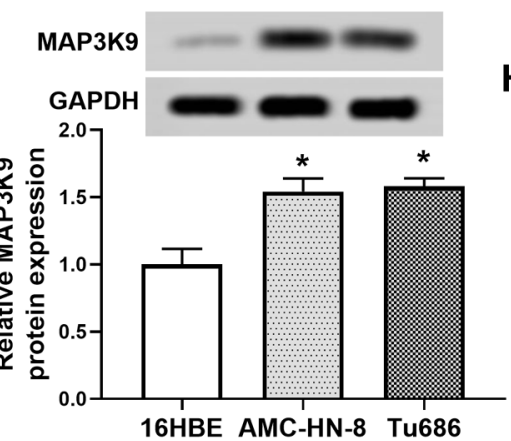

E

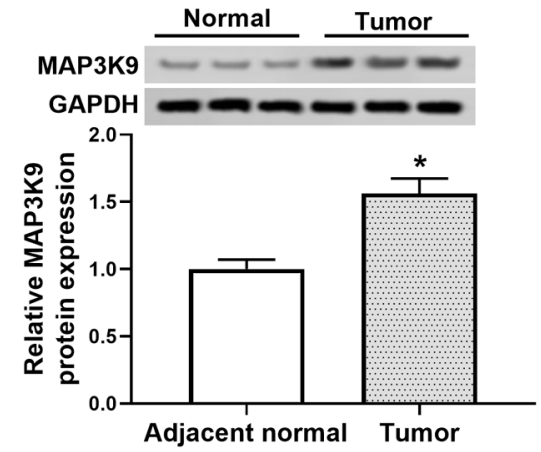

H

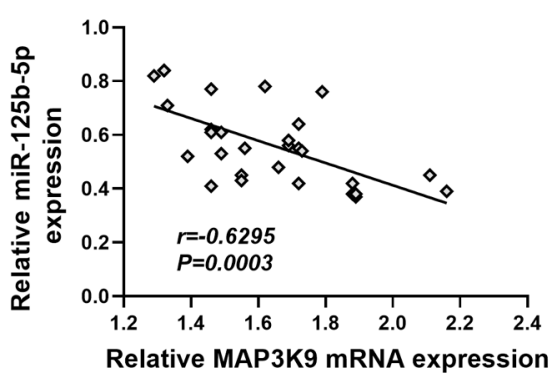

Figure 3. MAP3K9 was the direct target of miR-125b-5p. A) Diagram of the putative miR-125b-5p-binding site in the MAP3K9 3'-UTR was predicted by ENCORI (http://starbase.sysu.edu.cn/) and miRDB (http://mirdb.org/) websites. B) The effect of miR-125b-5p on luciferase activities of 3'-UTR-WT or 3'-UTR-MUT reporter was determined by luciferase assay kit. ${ }^{*} \mathbf{p}<0.05 \mathrm{vs}$. miR-NC. C) The effect of miR-125b-5p on luciferase activities of 3'-UTRWT or 3'-UTR-MUT reporter was determined by luciferase assay kit. ${ }^{*} \mathbf{p}<0.05$ vs. miR-NC. D) MAP3K9 mRNA expression was measured by qRT-PCR in LSCC tissues $(n=29)$ and normal tissues $(n=29)$. ${ }^{*} p<0.05$ vs. adjacent normal. E) MAP3K9 protein expression was measured by western blotting in LSCC tissues and normal tissues ${ }^{\star} \mathrm{p}<0.05$ vs. adjacent normal. F) MAP3K9 mRNA expression was measured by qRT-PCR in LSCC cells (AMC-HN-8 and Tu686 cells) and normal cells (16HBE). ${ }^{*} \mathrm{p}<0.05$ vs. $16 \mathrm{HBE}$. G) MAP3K9 protein expression was measured by western blotting in LSCC cells (AMCHN-8 and Tu686 cells) and normal cells $(16 \mathrm{HBE}) .{ }^{*} \mathrm{p}<0.05$ vs. $16 \mathrm{HBE}$. $\left.\mathrm{H}\right)$ The correlations between the expression of MAP3K9 and miR-125b-5p were analyzed by Spearman's rank correlation analysis.

HK2 expression were reversed by MAP3K9 overexpression (Figures 4G,4H). Overall, these results suggested that miR-125b-5p could inhibit the growth and glycolysis of LSCC cells via directly targeting MAP3K.

miR-125b-5p exerted cancer-inhibiting properties in tumor growth in vivo. Finally, we explored the effects of miR-125b-5p in vivo. Compared with the miR-NC group, the average tumor volume of the miR-125b-5p group significantly slowed down (Figure 5A). And the mean tumor weight of the miR-NC group was about twice as much as that of the miR-125b-5p group (Figure 5B). Additionally, the expression level of miR-125b-5p was upregulated in tumor tissues
(Figure 5C) and MAP3K9 expression was downregulated in tumor tissues by overexpressing miR-125b-5p (Figures 5D, 5E). Next, immunohistochemistry results showed that the increase of miR-125b-5p significantly suppressed cell proliferation-related protein Ki-67 (Figure 5F). TUNEL staining results demonstrated miR-125b-5p could effectively facilitate apoptosis (Figure 5G). Knockdown of miR-125b-5p has a promoting effect on tumor growth in vivo. As shown in Figures $5 \mathrm{H}$ and $5 \mathrm{I}$, miR-125b-5p knockdown reduced the tumor volume and size. Additionally, as shown in Figures 5J and 5K, miR-125b-5p knockdown downregulated the expression of miR-125b-5p and MAP3K9 compared with 
the untreated tumor tissues. Collectively, miR-125b-5p obviously inhibited LSCC progression through regulating MAP3K9 in vivo.

\section{Discussion}

The pathogenesis of LSCC has been a concern of an increasing number of researchers in recent years. Fu et al. found that miR-155 regulated LSCC cell proliferation and apoptosis through modulating p27Kip1 [23]. Rastogi et al. showed that suppression of miR-377 facilitated the migration and invasion of LSCC cells via regulating HDAC9 [24]. Additionally, Wang et al. demonstrated that miR-139-5p restrained the occurrence and progress of LSCC by modulating HOXA9 [25]. The above studies indicated that exploring the LSCC-associated miRNAs is of great significance to the clinical diagnosis and treatment of LSCC diseases. In the present study, miR-125b-5p expression was
A

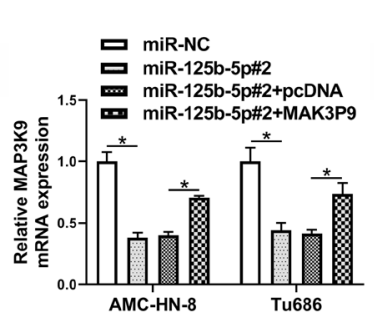

D

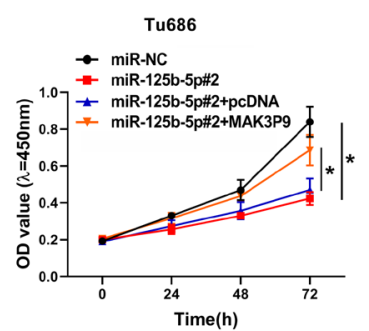

$\mathbf{F}$

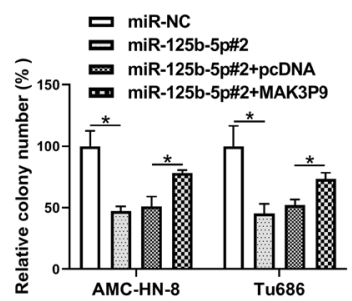

$\mathbf{H}$

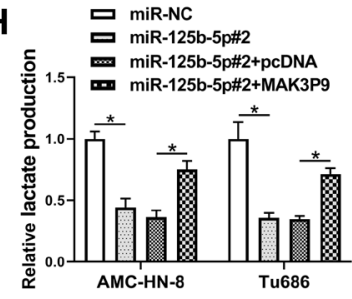

B
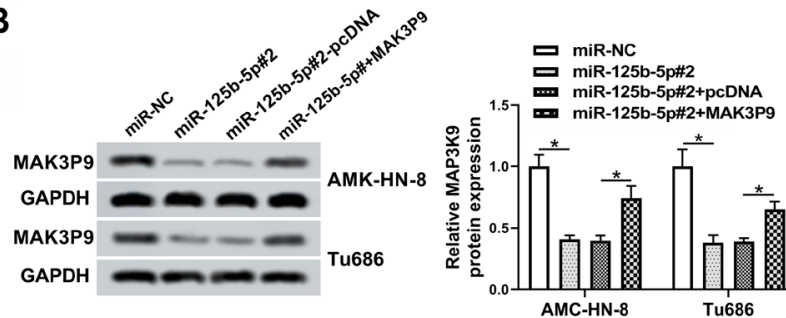

miR-125b-5p\#2 miR-125b-5p\#2
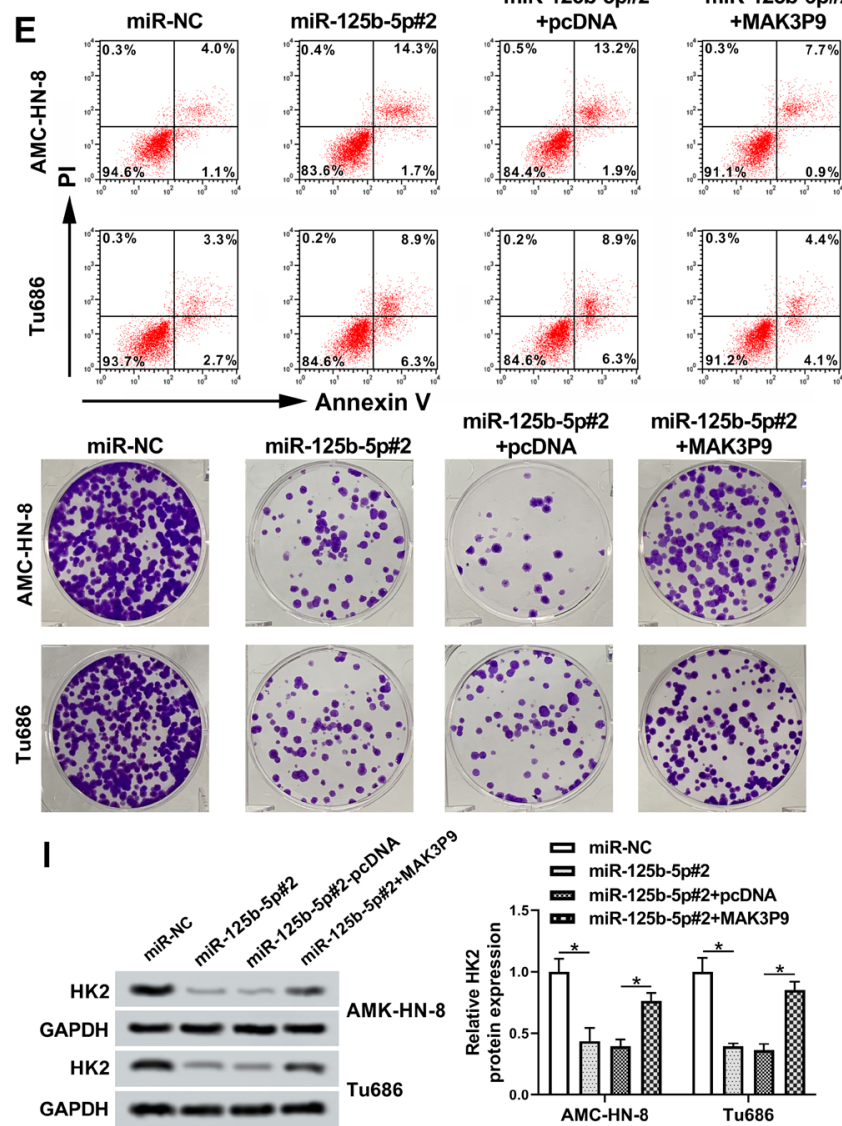

C
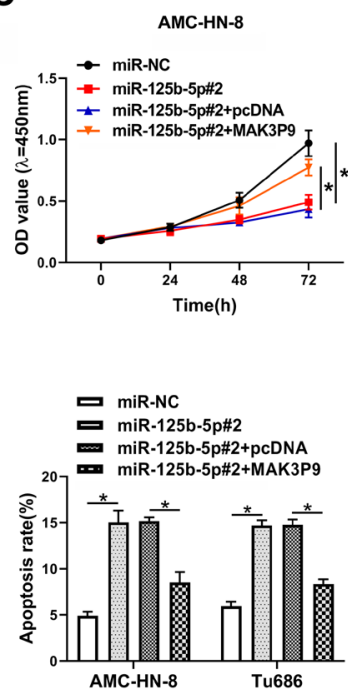

G

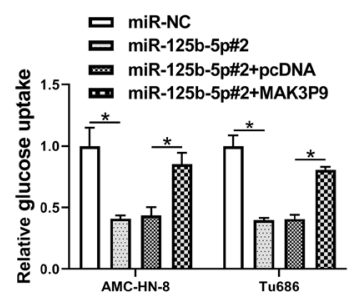

Figure 4. miR-125b-5p inhibited LSCC cell growth and glycolysis by downregulating MAP3K9. A) The efficiency of MAP3K9 overexpression was measured by qRT-PCR after $48 \mathrm{~h}$ of transfection. B) The efficiency of MAP3K 9 overexpression was measured by western blotting after $48 \mathrm{~h}$ of transfection. C) Cell viability was assessed by the CCK-8 assay in AMC-HN-8 cells co-transfected with miR-125b-5p\#2 and MAK3P9. D) Cell viability was assessed by the CCK-8 assay in Tu686 cells co-transfected with miR-125b-5p\#2 and MAK3P9. E) Apoptosis was detected by flow cytometry assay in AMC-HN-8 and Tu686 cells co-transfected with miR-125b-5p\#2 and MAK3P9. F) The relative number of clones was measured by colony formation assay in AMC-HN-8 and Tu686 cells co-transfected with miR-125b-5p\#2 and MAK3P9. G) Lactate production was measured by ELISA assay in AMCHN-8 and Tu686 cells co-transfected with miR-125b-5p\#2 and MAK3P9. H) Glucose uptake was measured by ELISA assay in AMC-HN-8 and Tu686 cells co-transfected with miR-125b-5p\#2 and MAK3P9. I) The expression of HK2 was measured by western blotting in AMC-HN-8 and Tu686 cells co-transfected with miR-125b-5p\#2 and MAK3P9. ${ }^{*} \mathrm{p}<0.05$. 
A

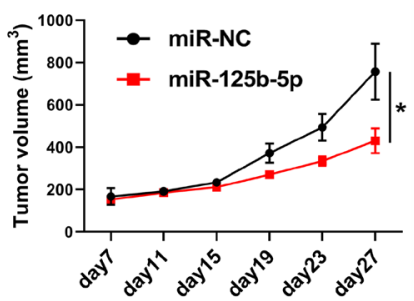

C

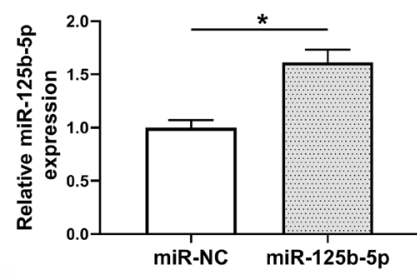

$\mathbf{F}$

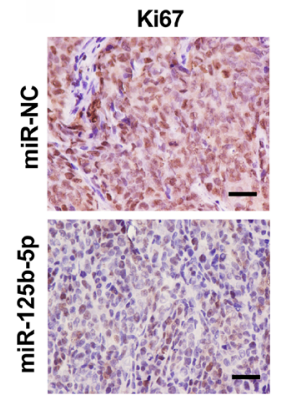

H
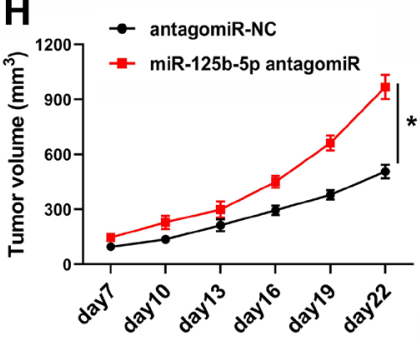

B

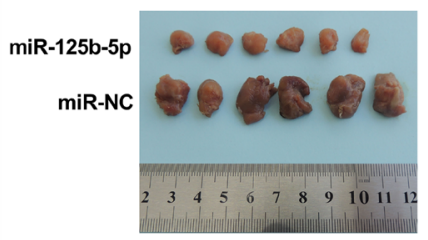

D

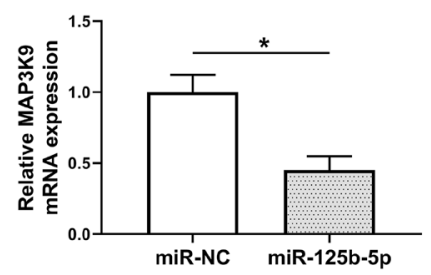

G

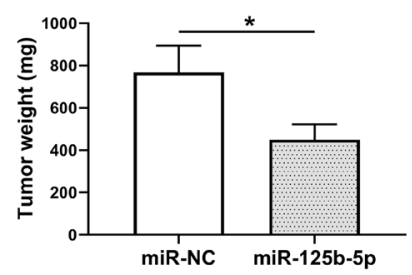

E

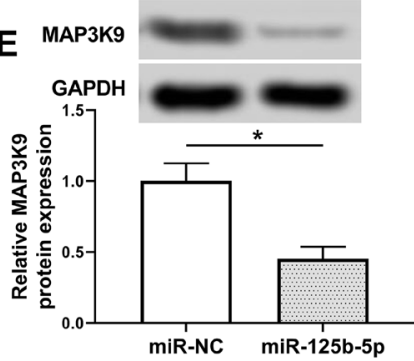

miR-NC miR-125b-5p

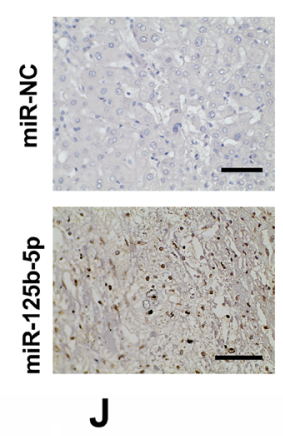

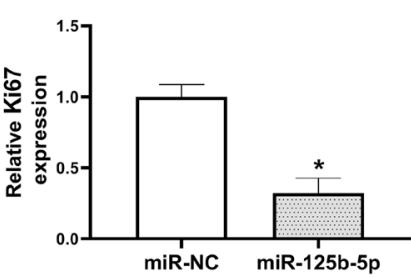

I
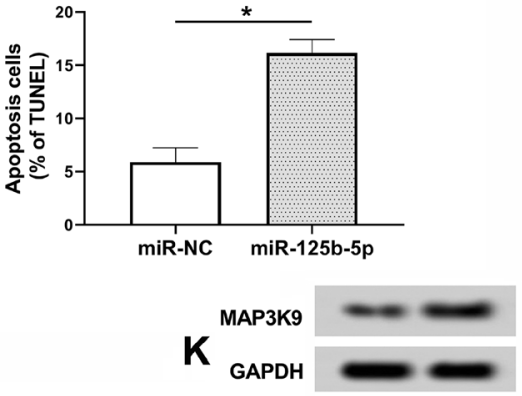
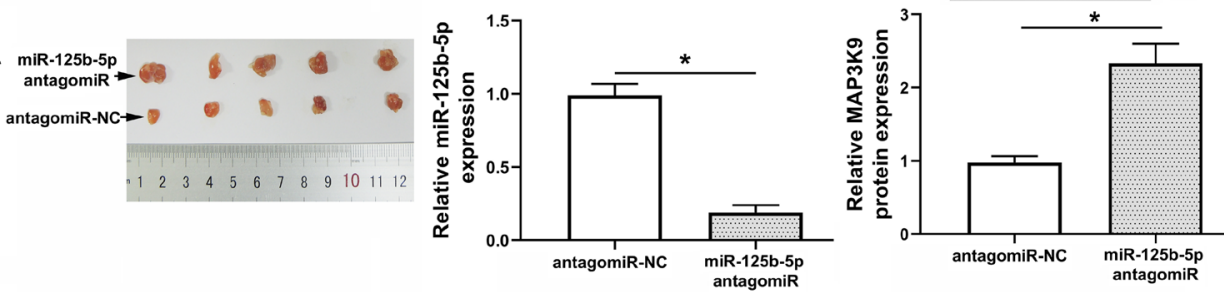

Figure 5. miR-125b-5p exerted cancer-inhibiting properties in tumor growth in vivo. A) Tumor volume in mice was monitored every third day for 4 weeks. Tumor growth curves were measured by the average volume of tumors for each group. ANOVA was performed to analyze significant values. ${ }^{*} \mathbf{p}<0.05$. B) Surgically removed tumor tissues from BALB/c mice of each group 27 days post-inoculation. The tumor weights of the two groups were measured 27 days post-inoculation. C) Expression of miR-125b-5p was determined by qRT-PCR in tumors of two groups. ${ }^{*} \mathbf{p}<0.05$. D) Effect of miR125b-5p overexpression on the mRNA expression level of MAP3K was measured by $q R T-P C R$ in tumors of two groups. ${ }^{*} p<0.05$. E) Effect of miR-125b$5 p$ overexpression on MAP3K9 protein expression was measured by western blotting in tumors of the two groups. ${ }^{*} p<0.05$. F) Proliferation-related protein (Ki-67) of tumor tissues from mice in each group was assessed by immunohistochemical staining. ${ }^{*} \mathbf{p}<0.05$ vs. miR-NC. G) Apoptosis was assessed by a TUNEL assay of tumor tissues from mice in each group. ${ }^{*} \mathbf{p}<\mathbf{0 . 0 5}$. $\mathrm{H}$ ) Tumor volume in mice was monitored every third day for $22 \mathrm{days}$. Tumor growth curves were measured by the average volume of tumors for each group. ANOVA was performed to analyze significant values. ${ }^{\star} \mathrm{p}<0.05$. I) Surgically removed tumor tissues from BALB/c mice of each group 22 days post-inoculation. The tumor weights of the two groups were measured22 days post-inoculation. J) Expression of miR-125b-5p was determined by qRT-PCR in tumors of two groups. ${ }^{*} \mathbf{p}<0.05$. K) Effect of miR-125b-5p knockdown on MAP3K9 protein expression was measured by western blotting in tumors of the two groups. ${ }^{*} \mathbf{p}<0.05$.

downregulated in LSCC tissues and cells. And miR-125b-5p could participate in the progression of LSCC and play an inhibitory role in LSCC by targeting MAP3K9.

miR-125b-5p was poorly expressed in major tumors, including $\mathrm{BC}, \mathrm{HCC}$, and malignant melanoma exerts a biological function in inhibiting cancer progression [16, $17,26]$. Now, Lian et al. proved that miR-125b-5p in LSCC cells was decreased, and the tumor cell progression could be inhibited by regulating glycolysis [18]. Subsequently, Liu et al. reported that IncRNA XIST promoted LSCC cell 
degeneration through sponging miR-125b-5p [22]. In recent research, miR-125b-5p was confirmed to be closely associated with tumor growth, clinical stage, and lymph node metastasis in patients with LSCC, suggesting the anti-tumor effect of miR-125b-5p in LSCC [27]. However, there are still few studies on miR-125b-5p in LSCC, and more studies are needed to clarify the different functions and regulatory mechanisms of miR-125b-5p in LSCC. In this study, the expression of miR-125b-5p was reduced in LSCC tissues and cells, and downregulation of miR-125b-5p could significantly prolong OS in patients. Moreover, miR-125b-5p overexpression effectively restrained the LSCC cell proliferation and induced apoptosis, and reduced the growth of tumor in vivo in mice. These results are consistent with the previous studies [22, 27]. In addition, glycolysis is the main energy-producing mode of tumor cells under the condition of adequate oxygen supply, and the abnormalities of lactate production and hexokinase (HK) are closely associated with the glycolysis pathway [28-30]. Notably, HK-2, as a member of the HK family, has been elevated in a variety of tumors and promoted glucose metabolism in tumor cells [31]. One previous research has shown that miR-125b-5p reduced LSCC cell glycolysis by inhibiting HK-2 expression, and then arrested cell growth [18]. In this study, consistent with the above research, miR-125b-5p overexpression repressed lactate production, glucose uptake, and HK-2 levels. These results further confirmed the suppressive role of miR-125b-5p on LSCC progression.

To understand the molecular mechanism of miR-125b-5p regulating LSCC and bioinformatics data predicted that MAP3K9 was the target gene of miR-125b-5p. MAP3K9, also known as MLK1, is also an upstream activator of mitogenactivated protein (MAPK) signaling, and its abnormal expression is closely related to the tumor progression $[32,33]$. In lung cancer, MAP $3 \mathrm{~K} 9$ mutations could positively activate the EMK/ERK pathway and then target to reduce the proliferation of lung cancer cells [34]. In cutaneous squamous cell carcinoma, miR-148a-3p repressed the invasion of tumor cells by downregulating MAP3K9 expression [35]. Interestingly, in pharyngolaryngeal cancer, overexpression of miR-490-5p repressed cell proliferation and metastasis through directly targeting MAP3K9, indicating the important regulatory role of MAP3K9 in laryngeal carcinoma [36]. However, no research has shown the function and concrete regulatory mechanism of MAP3K9 in LSCC. This study showed that MAP3K9 was upregulated in LSCC tissues and cells. Meanwhile, MAP3K9 was a target gene of miR-125b-5p, and their expression was negatively correlated. Overexpression of MAP3K9 could alleviate the inhibition of proliferation and glycolysis-related protein levels, and the promotion of apoptosis in miR-125b-5p overexpressing LSCC cells. To sum up, these results indicate that miR-125b-5p plays an anti-tumor effect in LSCC cells by targeting MAP3K9, which is characterized by inhibiting tumor cell proliferation and glycolysis, and inducing apoptosis.
However, there are still some limitations in this study. For instance, whether miR-125b-5p could reduce the progression of LSCC disease by regulating some classical pathways and the regulation of downstream target genes of MAP3K9 in LSCC needs to be investigated in subsequent experiments.

In conclusion, the current research showed that miR-125b-5p was lowly expressed in LSCC tissues and cells. High expression of miR-125b-5p was significantly associated with favorable OS in LSCC patients. miR-125b-5p effectively repressed the proliferation and glycolysis of LSCC cells and enhanced apoptosis by inhibiting MAP3K9. These data indicated that miR-125b-5p/MAP3K9 axis might be a potential diagnostic biomarker for LSCC treatment.

\section{References}

[1] BRAY F, FERLAY J, SOERJOMATARAM I, SIEGEL R, TORRE L et al. Global cancer statistics 2018: GLOBOCAN estimates of incidence and mortality worldwide for 36 cancers in 185 countries. CA Cancer J Clin 2018; 68: 394-424. https://doi.org//10.3322/caac.21492

[2] ALFOUZAN A. Head and neck cancer pathology: Old world versus new world disease. Niger J Clin Pract 2019; 22: 1-8. https://doi.org/10.4103/njcp.njcp_310_18

[3] CRAMER J, BURTNESS B, LE Q, FERRIS R. The changing therapeutic landscape of head and neck cancer. Nat Rev Clin Oncol 2019; 16: 669-683. https://doi.org/10.1038/s41571019-0227-Z

[4] SIEGEL R, MILLER K, JEMAL A. Cancer statistics, 2020. CA Cancer J Clin 2020; 70: 7-30. https://doi.org/10.3322/ caac. 21590

[5] LARBCHAROENSUB N, CHEEWARUANGROJ W, NITIYANANT P. Laryngeal sarcocystosis accompanying laryngeal squamous cell carcinoma: case report and literature review. Southeast Asian J Trop Med Public Health 2011; 42: 1072-1076.

[6] GAMEZ M, BLAKAJ A, ZOLLER W, BONOMI M, BLAKAJ $D$. Emerging concepts and novel strategies in radiation therapy for laryngeal cancer management. Cancers (Basel) 2020; 12: 1651. https://doi.org/10.3390/cancers12061651

[7] RUPAIMOOLE R, CALIN G, LOPEZ G, SOOD A. miRNA deregulation in cancer cells and the tumor microenvironment. Cancer Discov 2016; 6: 235-246. https://doi. org/10.1158/2159-8290.CD-15-0893

[8] ZHANG J, LIU J, LIU Y, WU W, LI X et al. MiR-101 represses lung cancer by inhibiting interaction of fibroblasts and cancer cells by down-regulating CXCL12. Biomed Pharmacother 2015; 74: 215-221. https://doi.org/10.1016/j.biopha.2015.08.013

[9] WANG G, DONG F, XU Z, SHARMA S, HU X et al. MicroRNA profile in HBV-induced infection and hepatocellular carcinoma. BMC Cancer 2017; 17: 805. https://doi.org/10.1186/ s12885-017-3816-1

[10] VISHNOI A, RANI S. miRNA biogenesis and regulation of diseases: an overview. Methods Mol Biol 2017; 1509: 1-10. https://doi.org/10.1007/978-1-4939-6524-3_1 
[11] WANG Y, CHEN M, TAO Z, HUA Q, CHEN S et al. Identification of predictive biomarkers for early diagnosis of larynx carcinoma based on microRNA expression data. Cancer Genet 2013; 206: 340-346. https://doi.org/10.1016/j.cancergen.2013.09.005

[12] SHUANG Y, ZHOU X, LI C, HUANG Y, ZHANG L. MicroRNA-503 serves an oncogenic role in laryngeal squamous cell carcinoma via targeting programmed cell death protein 4 . Mol Med Rep 2017; 16: 5249-5256. https://doi.org/10.3892/ mmr.2017.7278

[13] GAO W, WU Y, HE X, ZHANG C, ZHU M et al. MicroRNA204-5p inhibits invasion and metastasis of laryngeal squamous cell carcinoma by suppressing forkhead box C1. J Cancer 2017; 8: 2356-2368. https://doi.org/10.7150/jca.19470

[14] HUI L, ZHANG J, GUO X. MiR-125b-5p suppressed the glycolysis of laryngeal squamous cell carcinoma by downregulating hexokinase-2. Biomed Pharmacother 2018; 103: 1194-1201. https://doi.org/10.1016/j.biopha.2018.04.098

[15] SHEN Y, SHEN Z, GUO L, ZHANG Q, WANG Z et al. MiR$125 \mathrm{~b}-5 \mathrm{p}$ is involved in oxygen and glucose deprivation injury in PC-12 cells via CBS/H2S pathway. Nitric Oxide 2018; 78: 11-21. https://doi.org/10.1016/j.niox.2018.05.004

[16] LI Y, WANG Y, FAN H, ZHANG Z, LI N. MiR-125b-5p inhibits breast cancer cell proliferation, migration and invasion by targeting KIAA1522. Biochem Biophys Res Commun 2018; 504: 277-282. https://doi.org/10.1016/j.bbrc.2018.08.172

[17] HUA S, QUAN Y, ZHAN M, LIAO H, LI Y et al. MiR-125b$5 p$ inhibits cell proliferation, migration, and invasion in hepatocellular carcinoma via targeting TXNRD1. Cancer Cell Int 2019; 19: 203. https://doi.org/10.1186/s12935-0190919-6

[18] HUI L, ZHANG J, GUO X. MiR-125b-5p suppressed the glycolysis of laryngeal squamous cell carcinoma by downregulating hexokinase-2. Biomed Pharmacother 2018; 103: 1194-1201. https://doi.org/10.1016/j.biopha.2018.04.098

[19] LIVAK K, SCHMITTGEN T. Analysis of relative gene expression data using real-time quantitative PCR and the 2 (-Delta Delta C(T)) method. Methods 2001; 25: 402-408. https://doi.org/10.1006/meth.2001.1262

[20] HUANG L, LUO J, CAI Q, PAN Q, ZENG H et al. MicroRNA-125b suppresses the development of bladder cancer by targeting E2F3. Int J Cancer 2011; 128: 1758-1769. https:// doi.org/10.1002/ijc.25509

[21] FENG J, FAN Y, AYIHENG Q, ZHANG H, YONG J et al. MicroRNA-125b targeted STAT3 to inhibit laryngeal squamous cell carcinoma cell growth and motility. Oncol Lett 2017; 14: 480-486. https://doi.org/10.3892/ol.2017.6155

[22] LIU C, LU Z, LIU H, ZHUANG S, GUO P. LncRNA XIST promotes the progression of laryngeal squamous cell carcinoma via sponging miR-125b-5p to modulate TRIB2. Biosci Rep 2020; 40: BSR20193172. https://doi.org/10.1042/ BSR20193172

[23] FU S, CHEN H, CHENG P, ZHANG C, WU Y. MiR-155 regulates oral squamous cell carcinoma Tca8113 cell proliferation, cycle, and apoptosis via regulating p27Kip1. Eur Rev Med Pharmacol Sci 2017; 21: 937-944.
[24] RASTOGI B, KUMAR A, RAUT S, PANDA N, RATTAN V et al. Downregulation of miR-377 promotes oral squamous cell carcinoma growth and migration by targeting HDAC9. Cancer Invest 2017; 35: 152-162. https://doi.org/10.1080/07 357907.2017.1286669

[25] WANG K, JIN J, MA T, ZHAI H. MiR-139-5p inhibits the tumorigenesis and progression of oral squamous carcinoma cells by targeting HOXA9. J Cell Mol Med 2017; 21: 37303740. https://doi.org/10.1111/jcmm.13282

[26] LUAN W, DING Y, YUAN H, MA S, RUAN H et al. Long non-coding RNA LINC00520 promotes the proliferation and metastasis of malignant melanoma by inducing the miR125b-5p/EIF5A2 axis. J Exp Clin Cancer Res 2020; 39: 96. https://doi.org/10.1186/s13046-020-01599-7

[27] HUANG S, JIANG Y, YANG L, YANG J, LIANG $\mathrm{M}$ et al. Downregulation of miR-125b-5p and Its Prospective Molecular Mechanism in Lung Squamous Cell Carcinoma. Cancer Biother Radiopharm 2020; 7. https://doi.org/10.1089/ cbr.2020.3657

[28] JURISIC V, RADENKOVIC S, KONJEVIC G. The actual role of $\mathrm{LDH}$ as tumor marker, biochemical and clinical aspects. Adv Exp Med Biol 2015; 867: 115-124. https://doi. org/10.1007/978-94-017-7215-0_8

[29] TIAN L, ZHANG J, REN X, LIU X, GAO W et al. Overexpression of miR-26b decreases the cisplatin-resistance in laryngeal cancer by targeting ATF2. Oncotarget 2017; 8: 79023-79033. https://doi.org/10.18632/oncotarget.20784

[30] FITZPATRICK A, TUTT A. Controversial issues in the neoadjuvant treatment of triple-negative breast cancer. Ther Adv Med Oncol 2019; 11: 1758835919882581. https://doi. org/10.1177/1758835919882581

[31] BROECKER M, BECHER N, BOCKISCH A, DÜHRSEN U, MÜLLER S. Regulation of glucose uptake in lymphoma cell lines by c-MYC-and PI3K-dependent signaling pathways and impact of glycolytic pathways on cell viability. J Transl Med 2017; 15: 158. https://doi.org/10.1186/s12967-0171258-9

[32] LAI S, PELECH S. Regulatory roles of conserved phosphorylation sites in the activation T-loop of the MAP kinase ERK1. Mol Biol Cell 2016; 27: 1040-1050. https://doi.org/10.1091/ mbc.E15-07-0527

[33] SAMATAR A, POULIKAKOS P. Targeting RAS-ERK signalling in cancer: promises and challenges. Nat Rev Drug Discov 2014; 13: 928-942. https://doi.org//10.1038/nrd4281

[34] FAWDAR S, TROTTER E, LI Y, STEPHENSON N, HANKE $F$ et al. Targeted genetic dependency screen facilitates identification of actionable mutations in FGFR4, MAP3K9, and PAK5 in lung cancer. Proc Natl Acad Sci U S A 2013; 110: 12426-12431. https://doi.org/10.1073/pnas.1305207110

[35] LUO Q, LI W, ZHAO T, TIAN X, LIU Y et al. Role of miR$148 \mathrm{a}$ in cutaneous squamous cell carcinoma by repression of MAPK pathway. Arch Biochem Biophys 2015; 583: 47-54. https://doi.org/10.1016/j.abb.2015.07.022

[36] ABDEYRIM A, CHENG X, LIAN M, TAN Y. MiR-490-5p regulates the proliferation, migration, invasion and epithelial-mesenchymal transition of pharyngolaryngeal cancer cells by targeting mitogen-activated protein kinase kinasekinase 9. Int J Mol Med 2019; 44: 240-252. https://doi.org/10.3892/ ijmm.2019.4196 